\title{
A Hamiltonian Particle Method with Staggered Technique for Simulating Strong Ground Motion
}

\author{
Junichi TAKEKAWA ${ }^{1}$, Hitoshi MIKADA ${ }^{1}$ and Tada-nori GOTO ${ }^{1}$ \\ ${ }^{1}$ Dept. of Civil and Earth Res. Eng., Kyoto University
}

\begin{abstract}
We present a Hamiltonian particle method (HPM) with a staggered particle technique for simulating seismic wave propagation. In the conventional HPM, variables (e.g. displacement, stress) are defined at the same positions. On the other hand, most seismic simulations using finite difference methods (FDM) have used staggered grid techniques for improving the numerical accuracy. In the present study, we applied the staggered technique to HPM same as FDM. We applied our method to the conventional Lamb problem and compared numerical results of HPM with those from an analytical approach in order to demonstrate the effectiveness of the staggered particle technique. Our results showed better agreements with the analytical solutions than those from HPM without the staggered particles. This indicates that the staggered particle technique can be applicable to seismic wave simulations and improve the calculation accuracy.
\end{abstract}

\section{INTRODUCTION}

Seismic modelling techniques have been used for the predictions of strong ground motion induced by earthquakes ${ }^{1), 2), 3)}$ the investigations in rock physics 4), 5) and so on. Many numerical schemes have been developed in order to improve the numerical accuracy or efficiencies.

Finite difference method (FDM) has been widely used for its accuracy and simplicity in the seismological field. Virieux ${ }^{6), 7)}$ applied a staggered grid technique to simulate seismic wave propagation in order to improve the accuracy of FDM. In FDM with the staggered grid, the velocity and stress components are defined at different positions. And then, Saenger et al. ${ }^{8)}$ developed a rotated staggered grid to calculate the seismic wave propagation in arbitrary heterogeneous and anisotropic media. Since these techniques improve the accuracy without large increase of the computational costs, many researchers and engineers have used these techniques.

On the other hand, particle-based methods have also been applied to simulations of seismic wave propagation as an alternative to traditional continuum-based simulators. Toomey and Bean ${ }^{9)}$ developed an elastic lattice method (ELM) based on the distinct element method (DEM) ${ }^{10)}$. In ELM, particle velocities or displacements are defined at each particle position whereas interaction forces are defined between particles. Takekawa et al. ${ }^{11)}$ applied a Hamiltonian particle method (HPM), which was originally developed by Suzuki and
Koshizuka ${ }^{12)}$, to numerical simulations of seismic wave propagation induced by an earthquake. Their results showed the applicability of HPM to simulate seismic wave propagation on arbitrary free surfaces. In original HPM, however, the particle velocities or displacements and stress components are defined at the same particle positions. This induces an artificial oscillation which degrades the numerical accuracy. To overcome the disadvantage, Kondo et al. ${ }^{13)}$ developed a suppressing method through the introduction of an artificial force. Although their approach improved the accuracy of HPM dramatically, the additional calculations and storages for the artificial force are needed.

In the present study, we applied a staggered particle technique to HPM for seismic wave propagation in order to improve the accuracy and numerical efficiency. In our strategy, we define the displacement and stress components at the different positions similar to other simulators. First, we explain the basic theory of HPM with the staggered particles. Next, we test our numerical method using the conventional Lamb problem, and compare the results from HPM with the analytical solutions.

\section{METHOD}

In this chapter, we explain the basic theory of HPM and the staggered particle strategy. Figure 1 shows the arrangement of particles. Black and white circles represent main- and sub-particles. At the main-particles, the strain and stress tensors are defined. On the other hand, the displacement, 


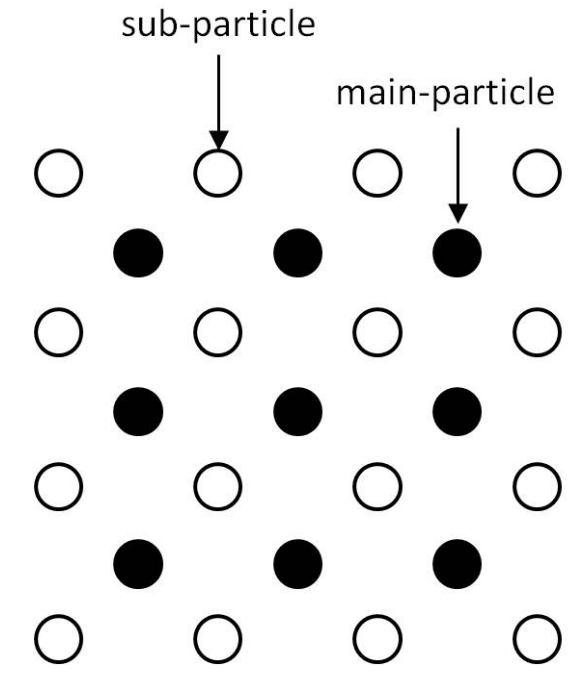

Figure 1: The staggered arrangement of the mainand sub-particles in the present study.

velocity and acceleration vectors are defined at the sub-particles. Each particle has an influence domain which defines the interacting particles around the particle. Interactions between particles described below are limited by the influence domain.

In HPM, the deformation gradient tensor is calculated by minimizing the error function $e_{i}$ as follows.

$e_{\mathrm{i}}=\sum_{\mathrm{j}}\left|\boldsymbol{F}_{\mathrm{i}} \boldsymbol{r}_{\mathrm{ij}}^{0}-\boldsymbol{r}_{\mathrm{ij}}\right|$

where $\mathbf{F}$ is the deformation gradient tensor, $\boldsymbol{r}_{\mathrm{ij}}^{0}$ and $\boldsymbol{r}_{\mathrm{ij}}$ are the initial and current position of sub-particle j relative to main-particle i, respectively. Subscripts $i$ and $j$ indicate main- and sub-particle, respectively. The summation in Eq.(1) is applied to the surrounding sub-particles inside the influence domain of main-particle i. Minimizing the error function derives the following equation for calculating the deformation gradient tensor.

$\boldsymbol{F}_{\mathrm{i}}=\sum_{\mathrm{j}} \boldsymbol{r}_{\mathrm{ij}} \otimes r_{\mathrm{ij}}^{0} A_{\mathrm{i}}^{-1}$

$\boldsymbol{A}_{\mathrm{i}}=\sum_{\mathrm{j}} \boldsymbol{r}_{\mathrm{ij}}^{0} \otimes \boldsymbol{r}_{\mathrm{ij}}^{0}$

$\mathrm{a} \otimes \mathrm{b}$ means tensor product of vector $\mathbf{a}$ and $\mathbf{b}$. The strain tensor, stress tensor and the total elastic strain energy can be calculated using the deformation gradient tensor.

$$
\begin{aligned}
& \boldsymbol{E}_{\mathrm{i}}=\frac{1}{2}\left(\boldsymbol{F}_{\mathrm{i}}^{\mathrm{T}} \boldsymbol{F}_{\mathrm{i}}-\boldsymbol{I}\right) \\
& \boldsymbol{S}_{\mathrm{i}}=2 \mu \boldsymbol{E}_{\mathrm{i}}+\lambda \operatorname{tr}\left(\boldsymbol{E}_{\mathrm{i}}\right) \boldsymbol{I} \\
& V=\sum_{\mathrm{i}} \frac{1}{2}\left(\boldsymbol{S}_{\mathrm{i}}: \boldsymbol{E}_{\mathrm{i}} \Delta \mathrm{B}_{\mathrm{i}}\right)
\end{aligned}
$$

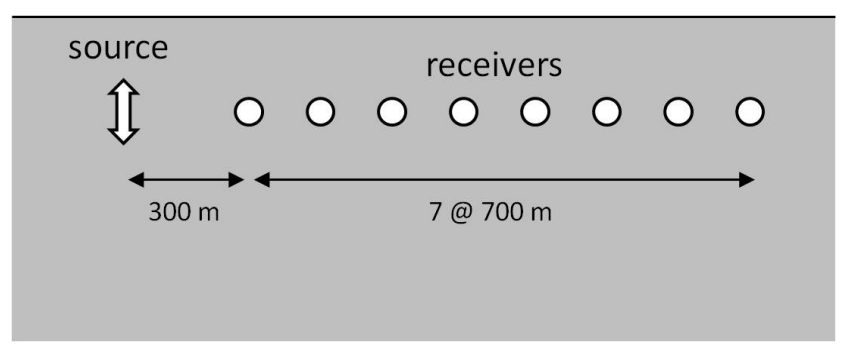

Figure 2: Schematic figure of our elastic half-space model.

where $\mathbf{E}, \mathbf{S}$ and $\mathrm{V}$ are the Green-Lagrangian strain tensor, second Piola-Kirchhoff stress tensor and the total elastic strain energy, respectively. $\Delta \mathrm{B}_{\mathrm{i}}$ is the volume of main-particle i. Using Hamiltonian's equation, we can derive the equation of motion for each sub-particle $j$.

$$
\begin{aligned}
\Delta \mathrm{m}_{\mathrm{j}} \frac{\partial v_{\mathrm{j}}}{\partial \mathrm{t}} & =\frac{\partial V}{\partial \boldsymbol{r}_{\mathrm{j}}} \\
& =\sum_{\mathrm{i}}\left(\boldsymbol{F}_{\mathrm{i}} \boldsymbol{S}_{\mathrm{i}} \boldsymbol{A}_{\mathrm{i}}^{-1} \boldsymbol{r}_{\mathrm{ji}}^{0} \Delta \mathrm{B}_{\mathrm{i}}\right)
\end{aligned}
$$

where $\Delta \mathrm{m}_{\mathrm{j}}$ is the mass of sub-particle $\mathrm{j}$. The summation in Eq.(7) is also applied to the surrounding main-particles inside the influence domain of sub-particle j.

\section{NUMERICAL RESULTS}

In this chapter, we conduct numerical simulations of surface wave propagation using the classic Lamb problem in order to demonstrate the effectiveness of our strategy. Figure 2 shows our numerical model of a half space. The seismic source and receivers are located at the depth of $100 \mathrm{~m}$. The source function is a Ricker wavelet with a central frequency of $4 \mathrm{~Hz}$, and the vertical force is applied at the source position. The receivers are set at equal distances from the seismic source from $300 \mathrm{~m}$ to $5200 \mathrm{~m}$. We locate the model boundaries well away from the source and receivers in order to avoid the artificial reflection waves instead of applying absorbing boundaries for the surrounding areas.

The spatial and time spacing are set to $10 \mathrm{~m}$ and $1 \mathrm{~ms}$, respectively. We studied two models A and B of an elastic homogeneous and isotropic medium. The model A has a P-wave velocity of $V_{p}=$ $4522 \mathrm{~m} / \mathrm{s}$, an S-wave velocity of $\mathrm{V}_{\mathrm{s}}=1846 \mathrm{~m} / \mathrm{s}$, and a mass density of $\rho=2200 \mathrm{~kg} / \mathrm{m}^{3}$. The model $\mathrm{B}$, on the other hand, has a P-wave velocity of $\mathrm{V}_{\mathrm{p}}=2611 \mathrm{~m} / \mathrm{s}$, an $\mathrm{S}$-wave velocity of $\mathrm{V}_{\mathrm{s}}=1846 \mathrm{~m} / \mathrm{s}$, and a mass density of $\rho=$ 
(a)
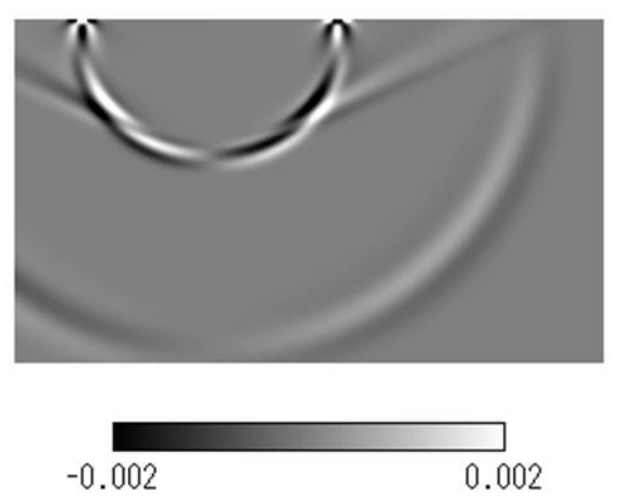

(b)

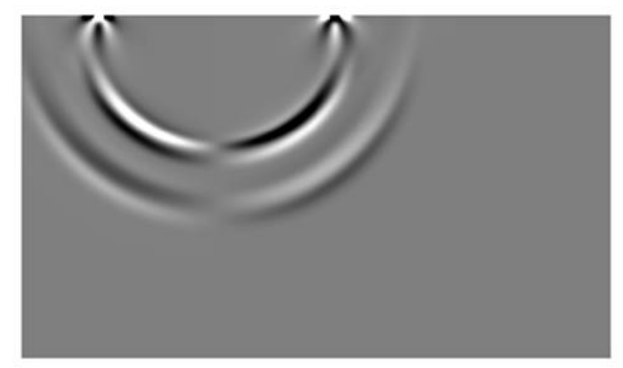

$-0.002$

0.002

Figure 3: The snapshots of the displacement field in the vertical direction after $2 \mathrm{~s}$ for (a) model $\mathrm{A}$, (b) model $\mathrm{B}$. The unit of the contour is meter.

$2200 \mathrm{~kg} / \mathrm{m}^{3}$. Model A and B have Poisson ratios of 0.4 and 0.0 , respectively.

We compare our numerical results with the analytical solution of Lamb problem, and evaluate the misfit by

misfit $=\frac{\sum_{\mathrm{t}}\left(\mathrm{s}^{\mathrm{NUM}}(\mathrm{t})-\mathrm{s}^{\mathrm{ANA}}(\mathrm{t})\right)^{2}}{\sum_{\mathrm{t}}\left(\mathrm{s}^{\mathrm{ANA}}(\mathrm{t})\right)^{2}}$

where $s^{N U M}(t)$ and $s^{A N A}(t)$ are the numerical and analytical seismograms, respectively.

Figure 3 shows the snapshots of the displacement field in the vertical direction calculated by HPM after $2 \mathrm{~s}$. In both models, we can observe the P, S and Rayleigh waves. Figure 4 shows the vertical displacement seismograms at each receiver. Solid and dotted lines are analytical and numerical seismograms, respectively. Thick broken lines are the differences between the seismograms amplified by a factor 5 . The misfits calculated by Eq.(8) are also shown at the right side of each seismogram. Both seismograms have good agreement with each other. At the farthest station, the misfits are less than 2 and $4 \%$ in models $A$ and B, respectively. Since the model B has slower velocity of the (a)
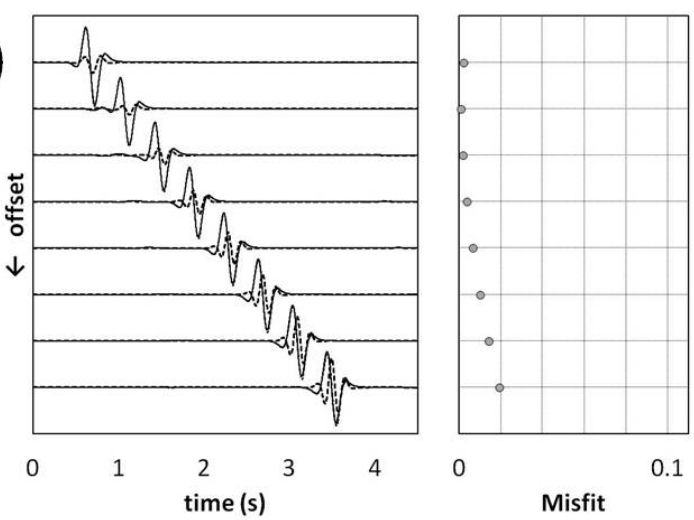

(b)
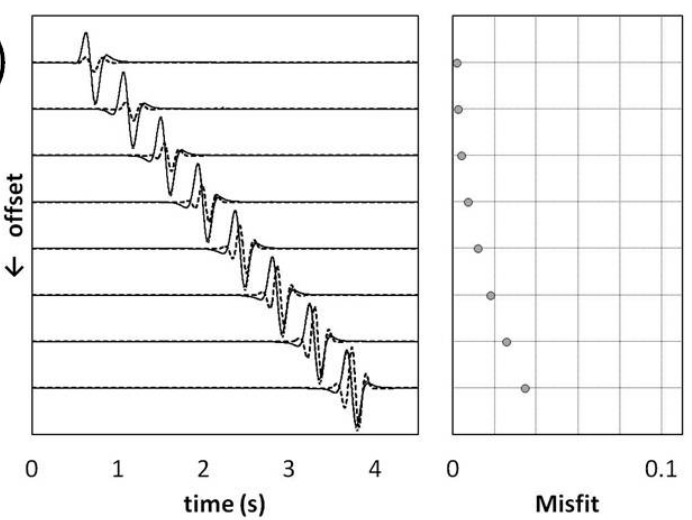

Figure 4: Vertical displacement at the receivers for (a) model A, (b) model B. Solid and dotted lines are the analytical and numerical seismograms, respectively. Dashed line is the differences between the seismograms amplified by a factor 5 . Gray circles right side of the seismograms represent the misfits calculated by Eq.(8).

Rayleigh wave than that of model A, the misfit becomes larger than that of model A. Anyhow, these results show better accuracy than those from HPM without the staggered particles ${ }^{11)}$.

\section{CONCLUSIONS}

In the present study, we applied the staggered particle technique to HPM for simulating seismic wave propagation. First, we explained our strategy to use staggered technique, and derived the governing equation for the particle motion. We, next, conducted surface wave propagation simulations to verify the effectiveness of our strategy by comparing with the analytical solutions. Numerical waveforms of HPM had good agreements with the analytical ones.

The application of the staggered particles cuts out the need of calculations for the artificial force. This decreases the numerical costs, e.g. calculation time and computational memory. Therefore, our strategy improves not only the accuracy of HPM but also the numerical efficiencies. 
ACKNOWLEDGMENT: This work was supported by MEXT/JSPS KAKENHI Grant Number 24760361.

\section{REFERENCES}

1) Graves, R. W., 1996, Simulating seismic wave propagation in 3D elastic media using staggered-grid finite differences, Bull. Seismol. Soc. Am., 86, 1091-1106.

2) Komatitsch, D. and Tromp, J., 1999, Introduction to the spectral element method for three-dimensional seismic wave propagation, Geophys. J. Int., 139, 806-822.

3) Koketsu, K., Fujiwara, H. and Ikegami, Y., 2004, Finite-element simulation of seismic ground motion with a voxel mesh, Pure Appl. Geophys., 161, 2183-2198.

4) Saenger, E. H. and Shapiro, S. A., 2002, Effective velocities in fractured media: a numerical study using the rotated staggered finite-difference grid, Geophys. Prospect., 50, 183-194.

5) Saenger, E. H., Kruger, O. S. and Shapiro, S. A., 2004, Effective elastic properties of randomly fractured soils: 3D numerical experiments: Geophys. Prospect., 52, 183-195.

6) Virieux, J., 1984, SH wave propagation in heterogeneous media: velocity-stress finite-difference method, Geophysics, 49, 1933-1957.

7) Virieux, J., 1986, P-SV wave propagation in heterogeneous media: velocity-stress finite difference method, Geophysics, 51, 889-901.

8) Saenger, E. H., Gold, N. and Shapiro, S. A., 2000, Modeling the propagation of elastic waves using a modified fnite-difference grid, Wave Motion, 31, 77-92.

9) Toomey, A. and Bean, C. J., 2000, Numerical simulation of seismic waves using a discrete particle scheme, Geophys. $J$. Int., 141, 595-604.

10) Cundall, P. A. and Strack, O. D. L., 1979, A discrete numerical model for granular assemblies, Geotechnique, 29, 47-65.

11) Takekawa, J., Madariaga, R., Mikada, H. and Goto, T., 2012, Numerical simulation of seismic wave propagation produced by earthquake by using a particle method, Geophys. J. Int., 191, 1305-1316.

12) Suzuki, Y. and Koshizuka, S., 2008, A Hamiltonian particle method for non-linear elastodynamics, Int. J. Numer. Methods Engng., 74, 1344-1373.

13) Kondo, M., Suzuki, Y. and Koshizuka, S., 2010, Suppressing local particle oscillations in the Hamiltonian particle method for elasticity, Int. J. Numer. Methods Engng., 81, 1514-1528. 